\title{
AVALIAÇÃO REPRODUTIVA E DESEMPENHO DA PROGÊNIE NA FASE INICIAL DE GALOS SEMIPESADOS COM DIFERENTES PESOS CORPORAIS
}

\author{
(Reproductive evaluation and performance of progeny in the initial phase of semi-heavy \\ cocks with different body weights)
}

Julmar da Costa Feijó, Frank George Guimarães Cruz, Ramon Duque Melo, João Paulo Ferreira Rufino, Emanuela Cristina De Souza Dias, Augusto Bruno Trindade Brandão ${ }^{2}$

\footnotetext{
1 Universidade Federal do Amazonas, Faculdade de Ciências Agrárias, Departamento de Produção Animal e Vegetal

${ }^{2}$ Universidade Federal do Amazonas, Instituto de Ciências Sociais, Educação e Zootecnia. Correspondência: augustoicsez@gmail.com
}

RESUMO: O objetivo deste estudo foi avaliar a capacidade reprodutiva e o desempenho da progênie (durante a fase inicial) de galos de duas linhagens semipesadas com diferentes faixas de peso. O delineamento experimental utilizado foi o inteiramente casualizado em esquema fatorial (3x2) constituído de dois fatores: três pesos corporais $(2,5 \mathrm{~kg}, 2,75 \mathrm{~kg}$ e $3 \mathrm{~kg})$ e duas linhagens semipesadas (Rhode Island Red e Plymouth Rock Barred), totalizando 6 tratamentos onde cada um possuía 5 galos analisados em três períodos de quatorze dias cada. Os dados coletados foram submetidos a análise de variância pelo teste de Tukey a $5 \%$ de significância. Galos mais pesados apresentaram melhores índices reprodutivos após a análise seminal, tendo a linhagem Rhode Island Red se sobressaído neste aspecto. Pintos oriundos de galos mais pesados apresentam maior peso ao nascer, sobressaindo novamente as aves da linhagem Rhode Island Red. Reprodutores machos mais pesados apresentam melhores índices reprodutivos seminais e de desempenho na sua progênie do que aves mais leves desde a análise da qualidade do sêmen, na relação entre o peso do ovo/peso do pinto e no desempenho da progênie na fase inicial do manejo. A linhagem Rhode Island Red apresentou melhores resultados para qualidade do sêmen do reprodutor e na relação peso do ovo de origem/peso do pinto, enquanto os pintos oriundos de galos da linhagem Plymouth Rock Barred apresentaram melhor desempenho durante a fase inicial.

Palavras-chave: andrológico; ganho de peso; motilidade; pintos; vigor

\begin{abstract}
The aimed of this study was evaluated the reproductive capacity and performance of the progeny (during the initial phase) of cocks of two brown lines with different weights. The experimental method was completely randomized design in factorial arrangement (3x2) with two factors: three body weight $(2.5 \mathrm{~kg}, 2.75 \mathrm{~kg}$ and $3 \mathrm{~kg})$ and semiheavy lineage (Rhode Island Red and Plymouth Barred Rock), totalling six treatments with five cocks analyzed in three periods of fourteen days each. The data collected were subjected to analysis of variance by Tukey test at $5 \%$ significance. Heavier cocks showed better reproductive rates after semen analysis, taking the lineage Rhode Island Red excelled in this aspect. Chicks from heavier cocks can have higher birth weight, highlighting strain again the birds of lineage Rhode Island Red. Heavier breeders showed better seminal reproductive rates and performance in their progeny than lighter breeders from the analysis of semen quality, the relationship between the egg weight/chick weight and performance of progeny in the management of early stage. The Rhode Island Red lineage showed better results for the breeder semen quality and ratio of origin egg weight/chick weight, while the chicks from cocks of Plymouth Barred Rock lineage showed better performance during the initial phase.
\end{abstract}

Key Words: andrological; weight gain, motility; chicks; vigour 


\section{INTRODUÇÃO}

O trabalho com as matrizes inicia o denominado ciclo da produção animal, sendo para a avicultura industrial, o ícone do galo 0 de maior representatividade por este ser o detentor do material genético repassado a progênie. $O$ reprodutor macho talvez seja o grande responsável para a manutenção dos índices de fertilidade, eclodibilidade, eclosão entre outras variáveis que demonstrem a evolução da produção, e para que ocorra um total aproveitamento da produtividade das matrizes, a determinação de um reprodutor ideal vai desde suas características fenotípicas e qualidade do seu sêmen até o potencial de seu material genético refletido na progênie, sugerindo assim que quaisquer alterações na produção ou na qualidade do sêmen dos machos podem gerar prejuízos tanto na produtividade da granja como na qualidade dos pintos produzidos (Maciel et al, 2011).

Dentre os fatores fisiológicos responsáveis por influenciar nos índices reprodutivos dos machos, destaca-se o peso corporal relacionado com o manejo alimentar apresentam-se com suma importância para 0 pleno desenvolvimento das funções reprodutivas. Conforme afirma Brake (1998), caso o galo não receba a quantidade de energia suficiente para sua manutenção, vai mobilizar suas reservas corporais; e se esta situação persistir e as reservas acabarem, haverá redução na atividade reprodutiva já que os níveis de testosterona irão cair drasticamente.

Outrora, Hocking \& Bernard (1997), em análises mais sucintas, evidenciaram que quando o peso corporal aumenta com a idade, ocorre um aumento no peso do músculo do peito, uma redução na frequência da atividade sexual, contribuindo com maior número de acasalamentos incompletos, afetando negativamente a fertilidade.

Além do reprodutor que irá transferir 0 potencial genético em questão, outro ponto a ser devidamente observado para que as matrizes se desenvolvam é o manejo na fase inicial, pois terá reflexos em quaisquer das fases subsequentes. Segundo Goulart et al. (2008), qualquer equívoco na nutrição de frangos na fase inicial, por exemplo, acarreta prejuízos econômicos posteriores.

A classificada fase inicial do manejo das matrizes inicia-se primordialmente no período póseclosão, quando o saco vitelino assume papel essencial nas primeiras 72 horas de vida do pinto e suas reservas nutricionais nesse período são consumidas. A velocidade dessa absorção do saco vitelino reflete também na maturação das funções digestivas e está relacionada com o início da aceitabilidade do alimento pela ave (Noy \& Sklan, 1996).

Sendo assim, as aves necessitam adquirir rápida capacidade de absorver nutrientes externos (Jin et al., 1998) e esse estímulo ao consumo do alimento sólido irá proporcionar alterações na estrutura física do aparelho digestivo e de suas secreções, indispensáveis para digestão dos nutrientes na fase inicial de vida. Tendo em vista a importância do manejo de matrizes na fase inicial e a possibilidade de caracteres produtivos dos galos para a progênie, realizou-se este trabalho com o objetivo de avaliar a capacidade reprodutiva e 0 desempenho da progênie (durante a fase inicial) de galos de duas linhagens semipesadas com diferentes faixas de peso.

\section{MATERIAL E MÉTODOS}

O experimento foi realizado nas instalações do Setor de Avicultura do 
Departamento de Produção Animal e Vegetal (DPAV) da Faculdade de Ciências Agrárias (FCA) da Universidade Federal do Amazonas (UFAM), Manaus/Amazonas.

Foram utilizados trinta galos semipesados com 53 semanas de idade, devidamente identificados e separados conforme a linhagem e a faixa de peso pré-estabelecida e alojados em galpão de modelo aberto com $8 \times 25 \mathrm{~m}^{2}$ de área sendo organizados em boxes $\left(9 \mathrm{~m}^{2}\right)$ com cama de maravalha. A fim de manter a homogeneidade, todas as aves utilizadas neste estudo foram submetidas à idênticas condições de manejo e alimentação, recebendo durante o período experimental 16 horas de luz/dia (12hs natural $+4 \mathrm{hs}$ artificial) e rações isonutritivas contendo $2800 \mathrm{Kcal}$ de $\mathrm{EM} / \mathrm{Kg}$ e $15 \%$ de $\mathrm{PB}, 4,0 \%$ de $\mathrm{Ca}$ e $0,45 \%$ de $\mathrm{Pd}$ segundo recomendações de Rostagno et al. (2011).

O delineamento experimental utilizado foi o inteiramente casualizado em esquema fatorial $(3 \times 2)$ constituído de dois fatores: três pesos corporais $(2,5 \mathrm{~kg}, 2,75 \mathrm{~kg}$ e $3 \mathrm{~kg})$ e duas linhagens semipesadas (Rhode Island Red e Plymouth Rock Barred), totalizando 6 tratamentos onde cada um possuía 5 galos analisados em três períodos de quatorze dias cada.

Inicialmente, os galos foram submetidos a exames andrológicos a fim de mensurar os índices reprodutivos e verificar o efeito do peso do galo e das linhagens sobre estes. As coletas de sêmen foram realizadas individualmente, totalizando 30 amostras (ejaculados) em cada período. O sêmen foi coletado pela técnica de massagem abdominal, segundo determina Bakst \& Bahr (1995), que constou de suave massagem no abdômen, movimentos rítmicos na sua parte ventral e nas penas da cauda do reprodutor. Quando a tumescência fálica foi atingida, as mãos do técnico eram colocadas ao redor da cloaca e os seus dedos polegar e indicador pressionavam levemente a cloaca ventralmente. As estruturas da cloaca não foram tocadas para evitar a disseminação de agentes patógenos. A coleta foi realizada pelo mesmo técnico para evitar variação nos resultados.

O volume foi determinado em seringa graduada $(1 \mathrm{~mL}, 13 \times 0,4 \mathrm{~mm})$ no momento da coleta, evitando assim que houvesse perdas. Imediatamente após a coleta, uma gota de sêmen foi colocada sobre lâmina e lamínula, sendo visualizada em microscópio óptico com aumento de 400x para avaliação das características dos espermatozoides. A motilidade foi determinada pela porcentagem de espermatozoides em movimento (0 à 100\%). O vigor foi estimado pelo movimento progressivo retilíneo e uniforme dos espermatozoides, em uma escala de zero a cinco, sendo o escore zero equivalente à ausência total de movimento dos espermatozoides e o escore cinco à movimentação intensa, vigorosa e progressiva.

A densidade foi mensurada a partir do escore de espaçamento entre os espermatozoides de zero a quatro, sendo o escore zero amplo espaço entre os espermatozoides e o escore quatro ausência total de espaço entre os espermatozoides. A textura foi atribuída à escala de zero a três, onde zero representou textura aquosa e três representou textura cremosa. A coloração foi acentuada em escores de zero a três, onde zero representou colocação branca aquosa e três representou coloração branca cremosa. Para determinação da concentração espermática, o sêmen foi diluído na proporção de 1:800 em solução de azul de metileno e os espermatozoides foram contados em câmara de Neubauer, sob microscopia óptica com aumento de 400x. 
corporais

Após a mensuração das análises andrológicas foi realizada nova retirada de sêmen para fins de inseminação artificial com intuito de avaliar 0 desempenho da progênie dos referidos galos durante sua fase inicial. Para isso foram recolhidas amostras de sêmen dos galos constituindo assim um pool de sêmen para cada tratamento, com o método de coleta do sêmen dos galos obedecendo à metodologia proposta anteriormente, utilizando a massagem abdominal no dorso e movimentos nas laterais da cloaca sendo o sêmen armazenado em frascos descartáveis.

Foram selecionadas para receber estes pools de sêmen quarenta e oito matrizes fêmeas semipesadas das respectivas linhagens utilizadas com 53 semanas de idade alojadas em gaiolas, sendo organizadas em seis blocos de quatro gaiolas (onde os blocos correspondiam aos tratamentos) com $1,0 \times 0,45 \times 0,50 \mathrm{~m}$ contendo oito aves em cada bloco de gaiolas. Os seis blocos foram separados em duas fileiras da seguinte maneira: três blocos de gaiolas na fileira direita com matrizes inseminadas com sêmen proveniente de galos com pesos médios de 2,5 kg, 2,75 $\mathrm{kg}$ e $3,0 \mathrm{~kg}$ da linhagem Rhode Island Red e três blocos de gaiolas na fileira esquerda com matrizes inseminadas com sêmen proveniente de galos com pesos médios de $2,5 \mathrm{~kg}, 2,75 \mathrm{~kg}$ e 3,0 $\mathrm{kg}$ da linhagem Plymouth Rock Barrada.

O procedimento utilizado para reversão da cloaca das fêmeas foi o proposto por Etches (1996), sendo o sêmen depositado diretamente no oviduto destas com o auxílio de uma pipeta de volume fixo a $0,025 \mathrm{~mL}$.

Foram selecionados 240 ovos aptos e incubáveis provenientes das matrizes inseminadas, onde foram separados quarenta ovos por tratamento a fim que estes originassem trinta pintos para avaliação da progênie em cada. A determinação deste número de pintos para avaliação da progênie foi feita mediante resultado de teste de incubação realizado com os galos que determinou a fertilidade e eclodibilidade média destes em $91,7 \%$ e $94 \%$, respectivamente, independente da linhagem.

Os ovos selecionados à medida que foram coletados, limpos e desinfetados até completarem sete dias de coleta, foram transportados para sala de armazenamento com temperatura de $18^{\circ} \mathrm{C}$ e umidade relativa de $74 \%$, respectivamente, a fim de manter o zero fisiológico e permanecendo nesta até o momento da incubação.

Após resfriamento, os ovos foram pesados e passaram por um préaquecimento de seis horas na sala de incubação antes de serem devidamente incubados. Foi utilizada incubadora modelo PETERSIME 168 regulada para manter constante a temperatura do bulbo seco em $37,6^{\circ} \mathrm{C}$ e umidade relativa de 60\%. Com 19 dias de incubação, foi realizada a ovoscopia e os ovos claros foram retirados para identificação dos inférteis ou dos embriões mortos. Os ovos, a seguir, foram transferidos para as bandejas de eclosão, as quais foram dispostas ao acaso em um nascedouro modelo PETERSIME 168 com termostato programado para manter a temperatura do bulbo seco em $36,6{ }^{\circ} \mathrm{C}$ e umidade relativa de $65 \%$. A retirada dos pintos do nascedouro ocorreu com 504 horas (21 dias) sendo separados trinta pintos eclodidos de cada tratamento para avaliação da referida progênie.

Os pintos selecionados foram pesados e transferidos para gaiolas com aquecimento automático tipo criadeira (uma para cada tratamento) contendo três compartimentos, onde cada compartimento recebeu dez pintos.

Estes permaneceram nas gaiolas durante seis semanas (fase inicial de manejo) e tiveram semanalmente avaliados seu desempenho. As variáveis avaliadas durante esse 
período foram: consumo de ração (consumo médio), ganho de peso (semanal), conversão alimentar (grama de ração por grama de peso ganho), peso corporal (após a fase inicial) e mortalidade (após a fase inicial).

Os dados coletados foram submetidos à análise de variância nos fatores individualmente e na interação entre estes, e para comparação das médias estimadas foi aplicado o teste de Tukey ao nível de $5 \%$ de probabilidade utilizando 0 procedimento GLM do programa computacional Statistical Analysis System - SAS (2008).

\section{RESULTADOS E DISCUSSÃO}

Os resultados para o exame andrológico estão expostos na Tabela 1. Foram observadas diferenças significativas $(P<0,05)$ na motilidade $e$ no vigor espermático entre pesos e linhagens, e na concentração espermática apenas entre linhagens. Os galos mais pesados apresentaram melhores resultados para motilidade, vigor e concentração, podendo indicar possível relação acerca do peso ideal para linhagens semipesadas em relação aos índices reprodutivos das matrizes (reprodutores machos) nestes.

Esta afirmação tende sobre esta possível sobreposição dos resultados principalmente devido em relação aos índices obtidos em galos com pesos corporais inferiores na motilidade e concentração, que segundo McDaniel et al. (1998), são os mais utilizados para determinar o potencial de fertilização de galos doadores de sêmen, já que estas variáveis influenciam o número total de espermatozóides disponíveis para fertilizar um ovo.

Além destes, um dos métodos para se estudar e quantificar esta eficiência reprodutiva em aves é estimar o número de espermatozoides que interagem com o oócito, no infundíbulo, como, por exemplo, o número de espermatozoides na membrana vitelínica externa, ou o número de buracos produzidos pelos espermatozoides na membrana vitelínica (Wishart, 1987; Hazary et al., 2000).

Todos estes, porém, atuam com o intuito de indicar ao técnico os melhores reprodutores, com melhor conformação corporal e habilidade sexual para fertilizar as fêmeas, conforme foi realizado neste estudo.

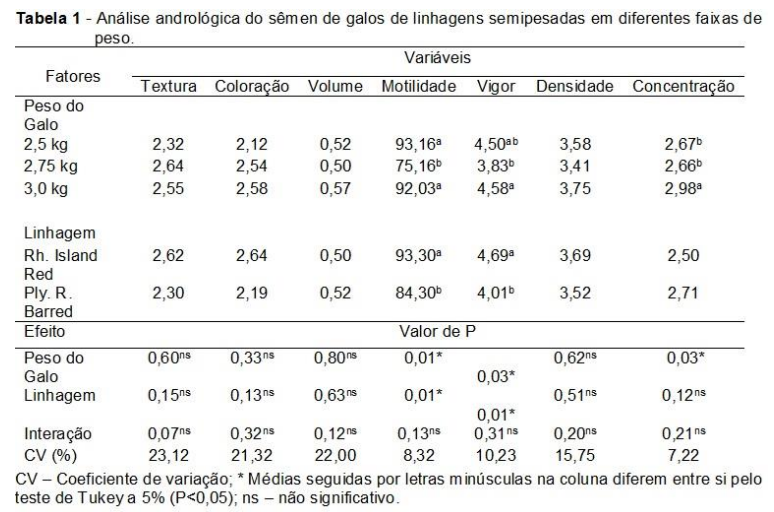

De acordo com os resultados encontrados para relação peso do ovo e peso do pinto (Tabela 2) foram observadas diferenças significativas $(P<0,05)$ apenas entre linhagens para peso do ovo, sendo que, apesar de ser um índice zootécnico passivelmente transmitido geneticamente, o peso do ovo relaciona-se mais a fatores nutricionais, ambientais e que influenciem as matrizes, 0 que notavelmente não distingue melhores resultados entre linhagens, porém, este resultado pode determinar que a presença reprodutiva do macho ou do sêmen (em casos inseminação) podem possuir relação com a característica reprodutiva das fêmeas com mesmo peso e linhagem.

Esta influência entre o ovo de origem e o peso do pinto à eclosão encontra-se bem documentada nas referências científica e os trabalhos mais atuais demonstram que o peso do pinto representa entre 66 a $71 \%$ do 
corporais

peso do ovo (Michalsky et al., 2005), sendo que o peso e a qualidade do pinto dependem de diversos vários fatores como a idade da matriz e o peso do ovo de origem, por exemplo (Peebles et al., 2000a), o que torna estudos desta categoria ferramentas que incrementam estes conhecimentos.

Com relação à qualidade do pintinho, esta pode ser avaliada através de diferentes métodos, sendo que cada um apresenta uma característica própria e depende do objetivo da avaliação em questão. Além disso, devem ser práticos, confiáveis, rápidos de executar e incluir características como o peso à eclosão (Hill, 2001; Wolanski et al., 2006) como instrumento de mensuração.

Contudo, a relação entre estes métodos de avaliação e o desempenho das aves, principalmente na fase inicial, ainda são pouco explorados. Sendo que ainda deve-se levar em consideração a influência exercida pelo galo reprodutor sobre o pintainho, o que levaria a análise tanto de desempenho da progênie quanto de correlações gênicas e testes laboratoriais.

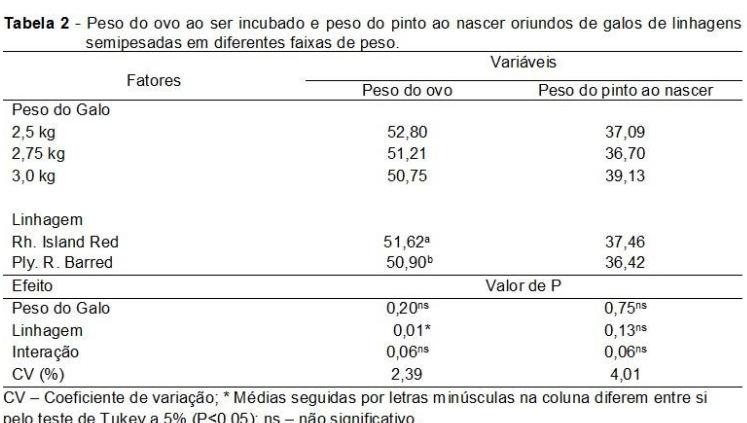

Os resultados para desempenho da progênie durante a fase inicial estão expostos na Tabela 3. Foram observadas diferenças significativas $(\mathrm{P}<0,05)$ para conversão alimentar, peso após a fase inicial e mortalidade entre pesos, e para peso após a fase inicial e mortalidade entre linhagens. A qualidade do pinto após a eclosão e com desempenho avaliado posteriormente pode ser utilizada como reflexo do padrão de manejo e genética de uma matriz e, também, das condições de desenvolvimento embrionário da ave durante a incubação (Rocha et al., 2008).

Verifica-se que pintos oriundos de galos mais pesados obtiveram melhores resultados, principalmente na relação entre a conversão alimentar e o peso após a fase inicial, onde apesar de possuírem maior conformação corporal e peso, obtiveram menor consumo de ração médio conseguindo mesmo assim transformar 0 alimento de forma eficiente em grama de peso vivo.

A eficiência de matrizes em diferentes fases pode ser influenciada de diversas formas e fatores, onde conforme Rufino et al. (2014) se destacam as instalações, os programas de iluminação, o manejo, a nutrição e a genética, onde estes influenciam diretamente a capacidade da matriz de expressar seu potencial.

Intencionalmente, por vários anos, o manejo de matrizes tem focado seu desenvolvimento principalmente no manejo nutricional associado ao desempenho para obter menores custos de produção e consequentes evoluções na qualidade dos pintos. Recentemente, diferentes trabalhos têm procurado avaliar 0 resultado deste manejo nutricional sobre o resultado final da progênie como os propostos por Peebles et al. (2002b) \& Araújo et al. (2009).

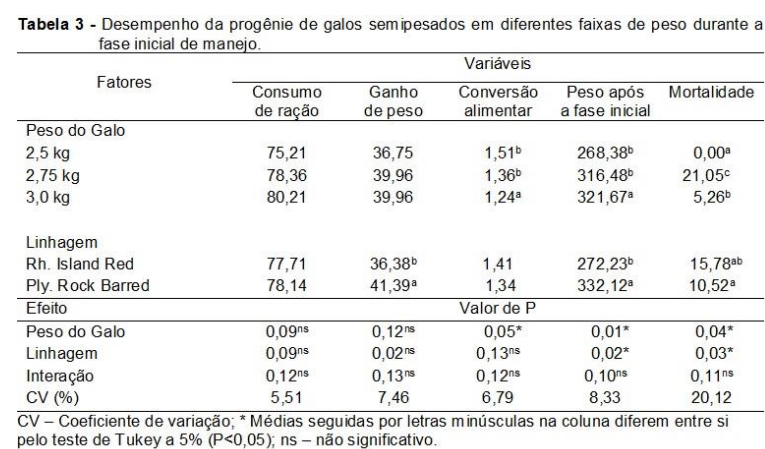

CV - Coeficiente de variaçẫo; ${ }^{*}$ Médias seguidas por letras
pelo teste de Tukey a $5 \%(P<0,05)$; ns - năo significativo. 
Entre linhagens, as aves oriundas de galos da linhagem Plymouth Rock Barred apresentaram índices superiores aos oriundos da linhagem Rhode Island Red, tendo demonstrado melhor capacidade de transformação do alimento consumido em peso vivo corporal. Atribuindo este conjunto de fatores a uma possível seleção genética, tem sido uma tendência do mercado 0 foco no potencial dos machos em transmitir os genes do ganho de peso cada vez mais acentuado (Robinson et al.,1993), o que implica em estudos com diferentes linhagens e seus caracteres genéticos. Neste caso, a linhagem pode ser considerada como um fator de influência nos resultados, pois há linhagens que apresentam maior habilidade reprodutiva do que outras, ou melhor capacidade de se adaptarem a um programa de manejo e melhoramento genético.

\section{CONCLUSÃO}

Reprodutores machos mais pesados apresentam melhores índices reprodutivos seminais e de desempenho na sua progênie do que aves mais leves desde a análise da qualidade do sêmen, na relação entre o peso do ovo/peso do pinto e no desempenho da progênie na fase inicial do manejo. Estas informações tornam-se imprescindíveis para o desenvolvimento de programas que busquem o melhoramento de um plantel de matrizes semipesadas, vertente ainda de pouca abrangência. A linhagem Rhode Island Red apresentou melhores resultados para qualidade do sêmen do reprodutor e na relação peso do ovo de origem/peso do pinto, enquanto os pintos oriundos de galos da linhagem Plymouth Rock Barred apresentaram melhor desempenho durante a fase inicial.

\section{NOTAS INFORMATIVAS}

O Comitê de Ética no Uso de Animais (CEUA) da Universidade Federal do Amazonas no dia 25 de janeiro de 2015 aprovou o projeto e recebeu o número 002/2015.

\section{REFERÊNCIAS}

ARAUJO, L.F.; ARAUJO, C.S.S.; ZUMWALT, C.D. et al. Impact of broiler breeder nutrition on progeny performance and carcass characteristics. International Scientific Forum, Anais (CD-ROM)... Atlanta, 2009.

BAKST, M.R.; BAHR, J.M. Ciclos reprodutivos: aves domésticas. In: HAFEZ, E. S. E. Reprodução animal. 6. ed. São Paulo: Manole, p. 390-407, 1995.

BRAKE, J. Equipment design for breeding flocks. Poultry Science, v.77, n.12, p.1833-1841, 1998.

ETCHES, R.J. Reproduction in poultry. Wallingford, UK: CAB International, 1996.

GOULART, C.C.; COSTA, F.G.P.; NETO, R.C.L. et al. Exigência de lisina digestível para frangos de corte machos de 1 a 42 dias de idade. Revista Brasileira de Zootecnia, v.37, n.6, p.878882, 2008.

HAZARY, R.C.; STAINES, H.J.; WISHART, G.J. Assessing the efficiency of mating in broiler breeder flocks by enumerating the spermatozoa which penetrate the inner perivitelline layer over the germinal disc. British Poultry Science, v.41, p.395-400, 2000.

HILL, D. Chick length uniformity profiles as a field measurement of chick quality? Avian and Poultry Biology Reviews, n.12, p.188, 2001.

HOCKING, P.M.; BERNARD, R. Effects of male body weight, strain and dietary protein content on fertility and muscle-skeletal disease in naturally mated broiler breeder males. British Poultry Science, v.38, n.1, p.29-37, 1997.

JIN, S.H.; CORLESS, A.; SELL, J.L. Digestive system development in posthatch poultry. World's Poultry Science Journal, v.54, p.335345, 1998.

McDANIEL, C.D.; HANNAH, J.L.; PARKER, H.M. et al. Use of a sperm analyzer for evaluating broiler breeder males. Effects of altering sperm quality and quantity on the sperm 
motility index. Poultry Science, v.77, n.6, p.888893, 1998.

MACIEL, M.P. Programas de luz sobre o desempenho e parâmetros seminais de galos semipesados. Ciência Rural, v.41, n.9, p.1617162, 2011.

MICHALSKY, V.B.; CANÇADO, S.V.; LARA, L.J.C. et al. Influência da umidade na incubação e idade da matriz leve sobre a eclosão e parâmetros de ovos e pintos. Brazilian Journal of Poultry Science, v.7, supl., p.13, 2005.

NOY, Y.; SKLAN, D. Routes of yolk utilization in the newly hatched chick. Poultry Science, v.75, n.59, p.13, 1996.

PEEBLES, E.D.; ZUMWALT, C.D.; DOYLE, S.M. et al. Effects of breeder age and dietary fat source and level on broiler breeder performance. Poultry Science, v.79, p.629-639, 2000.

PEEBLES, E.D.; ZUMWALT, C.D.; GERARD, P.D. et al. Market age live weight, carcass yield and liver characteristics of broiler offspring from breeder hens fed diets differing in fat and energy contents. Poultry Science, v.81, p.23-29, 2002.

ROBINSON, F.E.; WILSON, J.L.; YU, M.W. et al. The relationship between body weight and reproductive efficiency in mesttype chickens. Poultry Science, v.72, n.5, p.912-922, 1993.

ROCHA, J.S.R.; LARA, L.J.C.; BAIÃO, N.C. et al. Efeito da classificação dos ovos sobre o rendimento de incubação e os pesos do pinto e do saco vitelino. Arquivo Brasileiro de Medicina Veterinária e Zootecnia, v.60, n.4, p.979-986, 2008.

ROSTAGNO, H.S.; ALBINO, L.F.T.; DONZELE, J.L. et al. Tabelas brasileiras para aves e suínos: Composição dos alimentos e exigências nutricionais. $3^{\mathrm{a}}$ ed. Viçosa: Universidade Federal de Viçosa, 2011. 252 p.

RUFINO, J.P.F.; CRUZ, F.G.G.; MACHADO, N.J.B. et al. Processos de incubação artificial associados à aplicação de diferentes métodos reprodutivos em matrizes semipesadas. Revista Brasileira de Saúde e Produção Animal, v.15, n.3, p.765-773, 2014

Statistical Analysis System. SAS/STAT Software Version 9.2. Cary: SAS Institute Inc., 2008.

WISHART, G.J. Regulation of the length of the fertile period in the domestic fowl by numbers of oviducal spermatozoa. Journal of Reproduction and Fertility, v.80, p.493-498, 1987.

WOLANSKI, N.J.; RENEMA, R.A.; ROBINSON, F.E. et al. Relationship between chick conformation and quality measures with early growth traits in males of eight selected pure or commercial broiler breeder strains. Poultry Science, v.85, p.1490-1497, 2006. 